\title{
Quantitative assessment on public awareness of environmental protection in response to environmental incidents: a case for Taihu Lake algae bloom
}

\author{
L. Xu ${ }^{a}$, J. Shen ${ }^{b}$, D. Marinova ${ }^{a}$ and X. Guo ${ }^{a}$ \\ ${ }^{a}$ Curtin University Sustainability Policy (CUSP) Institute, Curtin University, Western Australia \\ ${ }^{b}$ School of Business, Hohai University, Nanjing 210098, P. R. China \\ Email: li.xu2@postgrad.curtin.edu.au
}

\begin{abstract}
In China, the water quality of most main lakes and catchments has been degrading since the 1970s. Specifically in 2009, large areas of the water in Huai, Hai and Liao rivers (three main rivers in China) and Taihu, Caohu and Dianchi lakes were of poor quality (below the worst category V), 17.4\%, 42.2\%, $36.1 \%$ 19.3\%, 62.5\% and 41.7\% respectively (China Environmental Protection Bureau, 2009). Furthermore, there is serious eutrophication in $85 \%$ of Chinese lakes with nonpoint sources pollution accounting for approximately $50 \%$ of the total pollutants discharge into the lakes in eastern China (Qin et al., 2007). Industrial discharges and household sewages are the main sources of water pollution in the country (Hays, 2008). While industrial pollution can be treated through technology renovation and controlled by environmental regulations and laws, decreasing household sewage in an efficient and sustainable way relies on the residents themselves. For example, more than $60 \%$ of the total phosphorus in Taihu Lake comes from household sewage and public awareness is essential for ensuring effective environmental improvement and protection (Chinese Academy for Environmental Planning, 2011).
\end{abstract}

Not many quantitative studies have been conducted to examine such environmental awareness, particularly the changes in the awareness levels in response to a major environment pollution incident. This paper establishes a system of indicators for the assessment of public awareness. Combined with a fuzzy matter-element analysis method, a model is set up to quantitatively assess public awareness of environmental protection. The model is applied to assess the environmental awareness of the residents of Wuxi city after their major lake Taihu suffered a severe algae bloom in 2007 which can be considered a serious environmental incident. This study aims at examining whether such an environmental incident can affect people's environmental awareness, and what is its degree of impact on the changes in public awareness of environmental protection in Wuxi. The study finds that the matter-element model is capable and resolves well the fuzziness of the ranking affiliation and classification for subjective objects such as people's environmental awareness. In addition, the assessment results indicate that the environmental incident has significant positive impacts on people's awareness of environmental protection. The paper concludes with some important recommendations related to raising people's environmental protection awareness.

References:

China Environmental Protection Bureau (2009). Communiqué: State of the Environment in China. http://www.instrument.com.cn/lib/editor/UploadFile/20106/20106483141246.pdf.

Chinese Academy for Environmental Planning (2011). Ten-Five Plan for the Prevention and Treatment of Taihu Lake. http://www.caep.org.cn/uploadfile/11-5/taihu.pdf (in Chinese).

Hays, J. (2008). Water pollution in China. http://factsanddetails.com/china.php?itemid=391\&catid=10\&sub catid $=66$

Qin, B., Z. Liu, and K. Havens (2007). Eutrophication of Shallow Lakes with Special Reference to Lake Taihu, China. Springer, Dordrecht, Germany.

Keywords: Fuzzy matter-element model, indicators system, Taihu Lake, Wuxi city, public environmental consciousness, impacts 


\section{INTRODUCTION}

Environmental problems are becoming increasingly severe in China (China Environmental Protection Bureau, 2009). The degradation of the environment has resulted in serious environmental disasters and incidents in the country and the algae bloomed in Taihu Lake in Wuxi city in the summer of 2007 is one of them. Between the end of May and the start of June in 2007, the algae bloomed and spread quickly into large areas of Taihu Lake - the third largest freshwater lake in China. The algae then contaminated all water intakes from the lake which is the water source for Wuxi city and also led to massive and rapid fish deaths. The water supply for Wuxi city was forced to be suspended for several days. During this period, the availability of drinking water was restricted, which resulted in hiking prices for fresh water and the whole city was full of chaos, rumors and panic (China Daily, 2007). Such environmental problems and situations are not only caused by contamination from industries and agriculture, but also instigated by poor public environmental awareness. Improving public awareness of environmental protection (PAEP) is the key measure for success in environmental protection (Yang, 2002). Although research on PAEP has been undertaken for over 40 years, very few quantitative studies have been conducted to examine such awareness, particularly in response to major environmental incidents. In this paper, a quantitative model is used to quantitatively assess PAEP and changes of PAEP in Wuxi after the algae bloom incident in Taihu Lake.

Owing to the complexity of the issues involved, most of the research conducted on assessing PAEP is based on subjective criteria, and a common system of indicators for evaluating PAEP is yet not available (Wang, 2000). The first series of systematic indicators called Ecological Attitudes and Knowledge Scale for assessing PAEP was established in 1975 by Maloney et al. (1975). It involved 4 different sub-scales and 45 items related to ecological knowledge, sensitivity, oral promises and real behaviours. Later on, the Environmental Concern Scale (Russel and Weigel, 1978) and New Environmental Paradigm Scale (Dunlap and Van Liere, 1978) were developed and used until the Value Scale put forward in 1992 by Schwartz's (1992) research group which suggested 53 sub-class values indicators derived from 10 kinds of human values. In 1994, Schwartz (1994) further classified the contents of the Value Scale into Self-transcendence, Self-enhancement, Openness and Tradition. Based on the individual value scale, Stern et al. (1995) centred their research on the primary motivation of individual environmental awareness and behaviours. They found that the motivation of PAEP and behaviours are mainly influenced by the degree of people's environmental values towards environmental protection, thus their environmental awareness and behaviours are dominantly influenced by biospheric values, social-altruistic values and egoistic values (Dunlap et al., 2000). Remarkably, the New Ecological Paradigm, set up and explored by Dunlap et al. (2000) after their New Environmental Paradigm Scale, is now a widely used indicator system. It includes 5 multi-faceted issues around the limits to growth, objection to anthropocentrism, fragility of the natural balance, humankind and any other species confined to natural ecosystems.

Many studies also focused on investigating and comparing PAEP in a certain area for a specific time (Kessel, 1985; Stabler et al., 1997; Huang et al., 2006; Liu et al., 2009). None of them are quantitatively mathematical but scoring assessment is used, particularly for the changes in PAEP in response to environmental pollution incidents. Quantitative assessment on the other hand can provide a very sharp and convincing warning about environmental problems. This paper therefore applies a fuzzy matter-element analysis method (Cai et al., 1997) to quantitatively assess PAEP and the 2007 algae bloom incident in Taihu Lake is used to quantify the changes in PAEP.

\section{INDICATOR SYSTEM AND MODELLING}

In this paper, PAEP is defined as a people's thinking pattern in reference to the environment and related issues, including their attitudes, social ideology, sensibility, environmental values, ethics, behavioural willingness and positivity of adjusting their socio-economic behaviour to natural requirements. The thinking pattern is imperceptibly generated and rooted in people's minds in the process of socio-economic development and material wealth creation. The assessment indicator system should be based on the above definition.

\subsection{Indicator System}

The indicator system is shown in Table 1 and comprises two assessment levels with 3 and 14 items respectively. 
Xu et al., Quantitative assessment on public awareness of environmental protection

Table 1. Indicators for the assessment of PAEP

\begin{tabular}{|c|c|c|}
\hline \multirow{15}{*}{$\begin{array}{c}\mathrm{P} \\
\mathrm{A} \\
\mathrm{E} \\
\mathrm{P} \\
(A)\end{array}$} & First Layer of Indicators & Second Layer of Indicators \\
\hline & \multirow{4}{*}{$\begin{array}{l}\text { Degree of familiarity with } \\
\text { the environment }\left(B_{1}\right)\end{array}$} & Degree of familiarity with the main environmental problems around $\left(C_{1}\right)$ \\
\hline & & Degree of familiarity with laws referring to the environmental protection $\left(C_{2}\right)$ \\
\hline & & Degree of familiarity with complaint lines about environmental offenders $\left(C_{3}\right)$ \\
\hline & & Degree of familiarity with basic environmental knowledge $\left(C_{4}\right)$ \\
\hline & \multirow{4}{*}{$\begin{array}{c}\text { Perceptual knowledge about } \\
\text { the environment }\left(B_{2}\right)\end{array}$} & Attitudes to pollution behaviour by industry plants and other people $\left(\mathrm{C}_{5}\right)$ \\
\hline & & Awareness of water use circulation $\left(\mathrm{C}_{6}\right)$ \\
\hline & & Attitudes to parts of views in the NEP scale $\left(\mathrm{C}_{7}\right)$ \\
\hline & & Attitudes to garbage sorting $\left(\mathrm{C}_{8}\right)$ \\
\hline & \multirow{6}{*}{$\begin{array}{l}\text { Rational knowledge about } \\
\text { the environment }\left(B_{3}\right)\end{array}$} & Willingness to pay for environmental protection $\left(C_{9}\right)$ \\
\hline & & Views about reasons for water use circulation $\left(C_{10}\right)$ \\
\hline & & Views about reasons for environmental degradation $\left(C_{11}\right)$ \\
\hline & & Views about obligations for environmental treatment $\left(C_{12}\right)$ \\
\hline & & Willingness for green consumption $\left(C_{13}\right)$ \\
\hline & & Willingness to think about environmental consequences before acting $\left(C_{14}\right)$ \\
\hline
\end{tabular}

\subsection{Quantitative Assessment Model}

Since PAEP is a kind of a subjective matter, parts of the indicators are fuzzy to assess. The PAEP assessment can be treated as a process of decision-making affected by multi-factors and a process of coping with contradicting problems from a quantitative study of qualitative indicators. Therefore the Matter-element Analysis method, a mathematical model developed by Cai in 1980s, is introduced and this is the first time for this method to be used in environmental social science. It aims to establish a multi-factor assessment model by using a ternary combination set $\boldsymbol{R}=(N, C, X)$ which synthesizes qualitative and quantitative indicators into a complex ternary combination set and consists of the assessment object $N$, its characteristics $\mathrm{C}$ and the corresponding values of each characteristic $\mathrm{X}$ so as to achieve the purpose of a quantitative assessment (Cai 1994; Cai et al., 1997). If an object $N$ is described by $\mathrm{n}$ characteristics $\left[c_{1}, c_{2}, \ldots, c_{\mathrm{n}}\right]$ with corresponding measures $\left[x_{1}, x_{2}, \ldots, x_{\mathrm{n}}\right]$, the n-dimensional mixed-element $\boldsymbol{R}$ can be described in a matrix as shown in equation (1):

$$
R=(N, C, X)=\left[\begin{array}{ccc}
N & c_{1} & x_{1} \\
& c_{2} & x_{2} \\
& \vdots & \vdots \\
& c_{n} & x_{n}
\end{array}\right]
$$

Accordingly, this mathematical modelling method is applied to the PAEP assessment as follows:

(1) Describe the assessment matter-elements

The first step for assessing PAEP using the matter-element method is to build up an $n$-dimensional matrix to describe the assessment object, including ranks and criterion of the assessment, on the basis of its characteristics and corresponding value of measures. In this study, there are 14 indicators for those selected characteristics (Table 1), i.e. $n=14$, thus the matrix is described as in (2):

$$
\left[\begin{array}{ccc}
N & c_{1} & x_{1} \\
& c_{2} & x_{2} \\
\vdots & \vdots \\
& c_{14} & x_{14}
\end{array}\right]
$$

where $x_{i}$ is the value for the measure of $c_{i}(i=1,2, \ldots, 14)$.

(2) Classical field and nodal field

A matrix for the classical field is then established using the follow equation (3): 
Xu et al., Quantitative assessment on public awareness of environmental protection

$$
R_{0}=\left[\begin{array}{ccc}
N_{0} & c_{1} & <a_{01}, b_{01}> \\
& c_{2} & <a_{02}, b_{02}> \\
& \vdots & \vdots \\
& c_{n} & <a_{0 n}, b_{0 n}>
\end{array}\right]
$$

where $N_{0}$ represents the condition of PAEP under a particular assessment rank. In this study, PAEP will be defined into four ranks (see Section 3 below). $C_{i}$ is the $i^{\text {th }}$ assessment indicator and $x_{0 i}=<a_{0 i}, b_{0 i}>$ is the value range for the measure of $C_{i}$; namely, $x_{\mathrm{oi}}$ is the value range (score space) of $C_{\mathrm{i}}$ under the assessment rank. This value range will be given by the scoring standard for each rank.

Similarly, the nodal field matrix for the evaluation of PAEP is developed and described as equation (4):

$$
R_{P}=\left[\begin{array}{ccc}
P & c_{1} & <a_{p 1}, b_{p 1}> \\
& c_{2} & <a_{p 2}, b_{p 2}> \\
\vdots & \vdots \\
& c_{n} & <a_{p n}, b_{p n}>
\end{array}\right]
$$

where $P$ indicates the collection of the whole ranks of the assessment criteria, $x_{p i}=<a_{p i}, b_{p i}>(i=1,2, \ldots, n)$ is the value range for all assessment indicators $c$, which is named as nodal field in the matter-element analysis, and $x_{0 i} \subset x_{p i}(i=1,2, \ldots, n)$.

(3) Develop the correlation function

The correlation function $K_{j}\left(x_{i}\right)$ in the model is used to describe the extent of each assessing indicator belonging to the ranks $j$ of the criteria. This correlation function is defined as equation (5):

$$
K_{j}\left(x_{i}\right)= \begin{cases}\frac{\rho\left(x_{i}, v_{0 i}\right)}{\rho\left(x_{i}, v_{p i}\right)-\rho\left(x_{i}, v_{0 i}\right)}, & x_{i} \notin v_{0 i} \\ -\frac{\rho\left(x_{i}, v_{0 i}\right)}{\left|v_{0 i}\right|}, & x_{i} \in v_{0 i}\end{cases}
$$

where $x_{i}$ is the value of indicator $i$ in the matter-element matrix being assessed while $\rho\left(x_{i}, v_{0 i}\right)$ and $\rho\left(x_{i}, v_{p i}\right)$ are the distances between point $x_{i}$ and classical field $v_{0 i}$ and nodal field $v_{p i}$ respectively with the distance of the field $|v|=|b-a|$, and

$$
\begin{aligned}
& \rho\left(x_{i}, v_{0 i}\right)=\left|x_{i}-\left(a_{0 i}+b_{0 i}\right) / 2\right|-\left(b_{0 i}-a_{0 i}\right) / 2 \\
& \rho\left(x_{i}, v_{p i}\right)=\left|x_{i}-\left(a_{p i}+b_{p i}\right) / 2\right|-\left(b_{p i}-a_{p i}\right) / 2
\end{aligned}
$$

(4) Calculate the correlation degree

According to the correlation function (5), the calculation for the integral correlation degree of object $N$ being assessed to rank $j$ is given by equation (6):

$$
K_{j}(N)=\sum_{i=1}^{n} w_{i} K_{j}\left(x_{i}\right),(i, j=1,2, \ldots)
$$

where $w_{i}$ indicates the standardized weight for the indicator $C_{i}$ of the being assessed object, $n$ is the quantity of indicators which is 14 in this study. If $K_{j 0}(N)=\max _{j \in\{1,2, \cdots, m\}} K_{j}(N)$, the conclusion could be made that the object $N$ being assessed belongs to rank $j_{0}$. This equation is used here to qualify the extent to which the PAEP condition complies with the criteria of rank $j$ for the considered indicator $x_{i}$ and not to derive statistical significance test. However it can calculate the value of the integral function $K$ for $x$ through another equation (not included in this paper), which can give a more precise status of the position of the indicators compared to the assessment ranks. 


\section{ASSESSMENT ON PAEP IN WUXI CITY AFTER THE TAIHU LAKE INCIDENT}

\subsection{Study Area}

Taihu Lake is the third largest freshwater lake in China and supports 34 million people, including Wuxi city in the Jiangsu Province, located in the core of the Yangtze River Delta and adjacent to Taihu Lake. The Taihu basin is situated in one of the most developed regions in China generating more than $10 \%$ of the country's GDP (Sun and Huang, 1993; Li et al., 2009). With the development of intensive industries discharging various chemicals and pollution from residents, the water quality of Taihu has declined from level 2-3 in 1990 to 4-5 in 2000 (World Bank, 2001). Water eutrophication and algae bloom have become serious environmental problems in this area.

In 2007, between the end of May and the beginning of June the algae bloomed in the lake and caused a crisis in the water supply to Wuxi. This serious incident impacted the local community and drew a lot of attention. The tap water supply in Wuxi was suspended for several days, and the whole city was full of chaos and panic (Anonymous, 2007). The reasons which caused water quality degradation in Taihu are multi-facets, including improper development of industrialization and low level of environmental awareness (Lin, 2002). In order to find effective ways to raise PAEP, it is important to examine what impact such an environmental incident has.

\subsection{Assessment Object and Indicators Ranking Criterion}

According to the indicators presented in Table 1 and equation (2), the assessment object can be described as a $14 \times 3$-dimensional matrix, $x_{i}$ is the score for $C_{i}$ and four classes are introduced to rank all those indicators: class I (bad), class II (medium), class III (good) and class IV (excellent). Correspondingly, the evaluation results are also divided into four classes: I (bad), II (medium), III (good) and IV (excellent). In addition, scores in the ranges of $[1,1.5],[1.5,2],[2,2.5]$ and $[2.5,3]$ are respectively assigned to these four classes. These four score ranges are the classical fields for the assessment indicators, and the nodal field therefore is $[1,3]$. The scoring criteria and descriptions of the indicators are available upon request from the authors.

\subsection{Weight System}

The Analytical Hierarchy Process (AHP) analysis (Satti, 1988) and expert scoring method are used to establish a weights system (Table 2).

Table 2. Weights and consistence test for PAEP assessment indicators system

\begin{tabular}{|c|c|c|c|c|c|c|c|c|c|c|c|c|c|c|}
\hline Indicators & \multicolumn{4}{|c|}{$B_{1}$} & \multicolumn{5}{|c|}{$B_{2}$} & \multicolumn{5}{|c|}{$B_{3}$} \\
\hline Weights & \multicolumn{4}{|c|}{0.251} & \multicolumn{5}{|c|}{0.159} & \multicolumn{5}{|c|}{0.589} \\
\hline \multicolumn{4}{|c|}{ Consistence Test } & \multicolumn{11}{|c|}{$\lambda_{\max }=3.053$, C.I. $=0.026$, C.R. $=0.045<0.10$} \\
\hline Indicators & $C_{l}$ & $C_{2}$ & $C_{3}$ & $C_{4}$ & $C_{5}$ & $C_{6}$ & $C_{7}$ & $C_{8}$ & $C_{9}$ & $C_{10}$ & $C_{11}$ & $C_{12}$ & $C_{13}$ & $C_{14}$ \\
\hline Weights & 0.029 & 0.018 & 0.018 & 0.036 & 0.023 & 0.041 & 0.055 & 0.047 & 0.119 & 0.081 & 0.101 & 0.137 & 0.138 & 0.156 \\
\hline \multicolumn{4}{|c|}{ Consistence Test } & \multicolumn{11}{|c|}{$\lambda_{\max }=14.645$, C.I. $=0.050, C . R .=0.031<0.10$} \\
\hline
\end{tabular}

\subsection{Data Collection}

According to our assumptions, the assessment of impacts of such an environmental incident can be treated as assessment of the PEAP level in different periods before and after the incident. A face-to-face survey was used to collect data and was conducted in Wuxi in August 2010 (two years after the algae bloom incident).

\subsection{Results and Discussion}

Using the scoring criteria and corresponding description with the results of weights and average scores for each indicator, the correlation function values (K) and classifications are calculated by equations (5) and (6).

Table 3 shows that the degree of PAEP in Wuxi has been generally growing. In particular, the improvement of PAEP started to accelerate after 2007 (the year of the Taihu Lake algae incident) and currently is at a medium and above level. Specifically, the max correlation function value of the indicators is -0.146 in the periods prior to the algae incident. The level of PAEP in Wuxi at this stage belongs to Class I and has about 0.129 distances from the smaller correlation function value of -0.275 which can be ranked as Class II. During the period of the algae incident, the max correlation function value for the assessment indicators for the level 
of PAEP is -0.190 which belongs to Class I. However the level of PAEP at this stage was higher than that in the period before the incident and the gap between Class I and Class II has narrowed down from 0.129 to 0.032 which means that the degree of PAEP during the incident went up by 0.097 . After the algae incident, at the time of the survey, the rank of PAEP in Wuxi has increased from Class I to Class II, and the correlation function value of rank is -0.123 . In addition, the rank of PAEP in this period is closer to Class III, and the gap between Class II and Class III is only 0.070 .

Table 3. Correlation values and assessment results of PAEP in Wuxi city

\begin{tabular}{|c|cccc|c|}
\hline \multirow{2}{*}{$\begin{array}{c}\text { Correlation Function } \\
\text { Values } K_{j}\left(N_{x}\right)\end{array}$} & \multicolumn{4}{|c|}{ Classifications/ Ranks $(j)$} & $j_{0}$ \\
\cline { 2 - 6 } & Bad (Class I) & Medium (Class II) & Good (Class III) & Excellent (Class IV) & I \\
\hline$K_{\text {before }}$ & -0.146 & -0.275 & -0.393 & -0.595 & I \\
\hline$K_{\text {during }}$ & -0.190 & -0.222 & -0.310 & -0.468 & II \\
\hline$K_{\text {after }}$ & -0.265 & -0.123 & -0.193 & & \\
\hline
\end{tabular}

These comparisons show that the degree of PAEP in Wuxi after the environmental incident is higher and growing faster than in the periods before and during the incident. It has increased by one rank since the incident.

\subsection{Recommendations for improvement of PAEP}

The assessment shows that the 2007 algae incident in Taihu Lake brought significant positive impacts on PAEP in Wuxi. To further raise the PAEP, more effective measures should be undertaken in the long term.

Firstly, there is a need to increase environmental education by using serious incidents as counter-examples. The impacts of the environmental incident in Taihu Lake on PAEP have been proven by our assessment results. Thus, it is important to emphasize the long-term education for environmental protection for the public by taking those environmental incidents as examples. Two ways are suggested: 1) organisations such as environmental agents, mass media and environmental non-government organisations (eNGOs) are responsible for making greater efforts to propagandize the serious impacts of environmental incidents by means of pictures and videos, which can alarm individuals through vivid scenes; 2) the harmfulness of the environmental incidents and its relationship with people's behaviours can be emphasized more during educational activities. As a result, public perceptual and rational knowledge about the environment $\left(B_{2}\right.$ and $\left.B_{3}\right)$ can be reinforced gradually.

Secondly, governments should set up long-term incentive systems for improving the PAEP. This study recommends that five steps should be put into the process as follows: 1) establish relevant regulations about incentive measures and corresponding criteria for those who have pro-environmental behaviour; 2) build up a governmental incentive fund for selected environmental activists in certain areas; 3) establish a regular intervening incentive time; and 4) publisize the shortlist of incentive holders via the mass media.

\section{CONCLUSIONS}

The main findings from the study are summarized below.

(1) The fuzzy matter-element method was introduced to establish a model for the assessment. It was found that the correlation function of the model is capable of resolving the fuzzy problems of the ranking affiliation and classification for the subjective assessing matters such as PAEP.

(2) It was found that such a major environmental incident like the algae bloom in Taihu Lake has significant impacts on PAEP and the experience obtained from the incident can be a key factor for improvement of PAEP.

(3) Recommendations to raise PAEP were made to increase environmental education by using such serious incidents as counter examples and establish long-term effective and incentive governance systems.

Due to the complexity of understanding PAEP and its influencing factors, further research on the long-term observations and series data information is required for better understanding of the development of PAEP.

\section{ACKNOWLEDGMENTS}

This study was funded by China's National Social Science Foundation (08DSH031) and is part of Xu's Master's thesis at Hohai University. The last two authors acknowledge the support of the Australian Research Council. 
Xu et al., Quantitative assessment on public awareness of environmental protection

\section{REFERENCES}

Anonymous (2007). Wuxi residents flee water crisis, China Herald, http://www.chinaherald.net/2007/06/ wuxi-residents-flee-water-crisis.html.

Cai, W., C.Y. Yang, and W.C. Lin (1997). Engineering Method of Extenics. Science Publication Press, Beijing (in Chinese).

Cai, W. (1994). Matter-element Model and its Application. Science and Technology Literature Publication Press, Beijing (in Chinese).

China Daily (2007). Blue-green algae bloom threatens water in Wuxi. http://www.newsgd.com/news/china1/200705310029.htm.

China Environmental Protection Bureau (2009). Communiqué: State of the Environment in China. http://www.instrument.com.cn/lib/editor/UploadFile/20106/20106483141246.pdf

Chinese Academy for Environmental Planning (2011). Ten-Five Plan for the Prevention and Treatment of Taihu Lake. http://www.caep.org.cn/uploadfile/11-5/taihu.pdf (in Chinese).

Dunlap, R.E., and K.D. Van Liere (1978). The new environmental paradigm: A proposed measuring instrument. Journal of Environmental Education, 9, 10-19.

Dunlap, R.E., K.D. Van Liere, A.G. Mertig, and R.E. Jones (2000). New trend in measuring environmental attitudes: Measuring endorsement of the new ecological paradigm: A revised NEP scale. Journal of Social Issues, 56 (3), 425-442.

Hays, J. (2008). Water pollution in China. http://factsanddetails.com/china.php?itemid=391\&catid=10\&sub catid $=66$

Huang, P., X. Zhang, and X. Deng (2006). Survey and analysis of public environmental awareness and performance in Ningbo, China: A case study on household electrical and electronic equipment. Journal of Cleaner Production, 14(18), 1635-1643.

Kessel, H. (1985). Changes in environmental awareness: A comparative study of the FRG, England and the USA. Land Use Policy, 2(2), 103-113.

Li, Y.K., Y. Chen, B. Song, D. Olson, N. Yu, and L.Q. Chen (2009). Ecosystem structure and functioning of Lake Taihu (China) and the impacts of fishing. Fisheries Research, 95(2-3), 309-324.

Lin, Z. (2002). Analysis of water environmental change in Taihu watershed. Jo Lake Sci., 14(2), 116-123.

Liu, Q., H. Li, X. Zuo, F. Zhang, and L. Wang (2009). A survey and analysis on public awareness and performance for promoting circular economy in China: A case study from Tianjin. Journal of Cleaner Production, 17(2), 265-270.

Maloney, M.P., M.P. Ward, and G.N. Braucht (1975). A revised scale for the measurement of ecological attitude and knowledge. American Psychologist, 30, 787-790.

Qin, B., Z. Liu, and K. Havens (2007). Eutrophication of Shallow Lakes with Special Reference to Lake Taihu, China. Springer, Dordrecht, Germany.

Russell, W.H., and J. Weigel (1978). Environmental concern: The development of measure. Environment and Behaviour, 10(1), 3-15.

Satti, T.L. (1988). The Analytical Hierarchy Process: Application in Distribution, Management and Conflict of Resource. Coal and carbon industry publication Press, Beijing: (in Chinese).

Schwartz, S. H. (1992). The universal content and structure of values: Theoretical advances and empirical tests in 20 countries. Advances in Experimental Social Psychology, 25, 1-62.

Schwartz, S.H. (1994). Are there universal aspects in the structure and contents of human value? Journal of Social Issues, 50(4), 19-45.

Stabler, M.J., and B. Goodall (1997). Environmental awareness, action and performance in Guernsey hospitality sector. Tourism Management, 18(1), 19-33.

Stern, P.C., T. Dietz, L. Kalof, and G.A. Guagnano (1995). Values, beliefs, and proenvironmental attitude formation toward emergent attitude objects. Journal of Applied Social Psychology, 25, 1611-1636.

Sun. S., and Y. Huang (1993). Taihu Lake. China Ocean Publication Press, Beijing (in Chinese).

Wang, M. (2000). The generation and definition of the concept of environmental awareness (in Chinese). Journal of Dialectics of Nature, 128(4), 86-90.

World Bank (2001). A Strategic Water Quality Improvement Plan for Thai Lake. http://www.socialassessment.com/akdocs.html.

Yang, M. (2002). Environment Issues: Awareness and Perceptions. Huaxia Press, Beijing (in Chinese). 